\title{
A Writing Kit for Engineering Design Reports
}

\author{
Margaret Hundleby \\ Writing Services \\ University of Guelph \\ hundleby@uoguelph.ca
}

\author{
Medhat Moussa \\ School of Engineering \\ University of Guelph \\ mmoussa@uoguelph.ca \\ Peggy Pritchard \\ McLaughlin Library \\ University of Guelph \\ ppritcha@uoguelph.ca
}

\author{
William David Lubitz \\ School of Engineering \\ University of Guelph \\ wlubitz@uoguelph.ca
}

\begin{abstract}
Targeted writing instruction is introduced into a third year design course in a series of four required courses in engineering design at the University of Guelph. A variety of writing resources are gathered and adapted to the needs of students required to deliver a 40-page Design Report for a client-based project. The Writing Kit uses a multi-layered approach, including online and print resources and composing workshops explaining writing for analytic purposes, and makes rubrics available for self-evaluation by student writers. Instruction focuses on the relationship of written documentation to design work and the importance of outcomes for evaluating the success of the work as well as for producing it. The writing kits is available for download from the University of Guelph library repository http://atrium.lib.uoguelph.cal
\end{abstract}

\section{Introduction}

A pilot project for introducing writing instruction into the four-course design sequence taken by all engineering students at the University of Guelph was mounted in Winter 2010 as part of Engineering and Design III (ENGG*3100). As the third course in the sequence, ENGG*3100 is centered on the completion of a semester-long open-ended design project by groups of three to four students.

All of the projects are based on a problem identified by an external sponsor, who varies from local industrial firms to international non-government organizations working overseas. The primary learning objectives are to continue to reinforce design skills taught in the prior courses in the sequence; develop skills in research and the integration and application of knowledge; and reinforcing principles of engineering communica- tion, with an emphasis on technical writing.

The initial impetus for integrating writing instruction comes from the belief of the course instructors that improvement of writing skills consistently needed as part of fully effective Engineering Education. While it is normal to have dedicated technical writers in large companies, their main job is to assist engineers in creating the documentation for a project rather than originating it. They often don't even exist in in small to medium firms, where engineers must take full responsibility for reporting on and explaining their work to colleagues, clients, and users. Similar responsibility often falls on students while in school, where they are routinely asked to write reports to document their design or lab. These are usually short papers of about ten pages, reflecting the necessarily limited focus and nature of the project/lab.

In ENGG 3100, however, we have undertaken to develop structured writing skills that can be used for authoring both long or short documents. Students are required to submit four documents, two short and two long. The first is a project proposal which is due in the second week of the twelve week term. The document identifies the members of the group and the project the group plans to work on, providing additional information on the method for analysing the problem and generating a design solution. Information is based on an initial investigation that includes a brief site visit and some limited research. This assignment aims at production of a short document that places the project and the work required for it in context.

In Week 6, all groups are required to submit the first of two major project reports, with the second report is due in Week 12. The page limit of 40 pages, plus appendices, moves students into a realm of expanded research and writing demands. At the same time, it requires stu- 
dents to increase their efforts to focus and control the content of the design report that forms a key deliverable for the project.

The fourth documentation requirement falls in the final week of the course. It comprises a two-page, IEEEstyle paper that documents the group design. This paper is reviewed, revised, and then collected as "Proceedings" deposited in the university's library and also made available from the on-line repository, Atrium.

To help students meet the documentary requirements and improve their skills for technical communication in the specific context of a design project, we developed a writing kit. This kit includes tools to insure a systematic approach to expanding skills and producing consistent, usable documentation. It encourages moving away from a standard, generalized undergraduate response to writing assignments to work on developing skills Through a series of research and writing workshops, supported by "how to" information and freely available consultation, students are helped to understand how to approach and evaluate their writing. Additionally, a marking rubric for the design reports is provided to students to help them with peer review of their reports before they deliver them for instructor evaluation.

The main contributors to this writing kit are the four authors of this paper. Margaret Hundleby is a professional technical writer who has longterm experience in helping engineers enhance their writing skills, especially in a design context. Medhat Moussa and Dave Lubitz have been the instructors of ENGG*3100 for the last three years and are responsible for the conception and implementation of the entire kit. Peggy Pritchard helped to guide students through the demands of indepth research and documentation, using the University of Guelph Library.

\section{Writing Kit Design}

\subsection{Major components}

This kit consists of three parts. The first part is a LaTeX package that includes a report template and a "getting started" file that point students to sources for a LaTeX editor and help documents. A popular, free typesetting program that is commonly used in scientific publication circles, including this conference proceeding, LaTeX has the key benefit of an emphasis on uniform formats and consistent structure for long documents. Students need only to focus on content not how to set up the document. No group member has to worry about the others using different fonts or style formats to author their parts. Built-in cross-referencing to the various parts of the text makes it easy to re-format and revise. The results are professional-looking documents with standard formats and style.
The second part of the kit consists of detailed instructions on how to organize the technical content from the project with the objective of composing a comprehensive design report. This part of the kit features explanation of the techniques for defining section content, developing informative paragraphs, and writing focussed sentences. The instruction is delivered in two ways: it is embedded in the LaTeX template as comments, so that students writing in the file can read them as they work, without their appearing when the document is printed; print resources are also provided in the form of charts and explanatory PowerPoint slides keyed to composing a specific document.

The third part of the kit consists of the rubrics used to mark the documents. They served initially as a source for the support materials, but also are coordinated with the writing instruction the students received to define specifically the expectations students are expected to fulfill. These rubrics are provided to students before they submit their report to help them evaluate their work.

\subsection{Theoretical basis of the kit}

The Writing Kit for Engineering Design Reports consists of four sets of materials designed to support the writing assignments of ENGG*3100 - an on-line LaTeX template, guidelines for managing data and composing specific genre, PowerPoint handouts containing the information from in-class writing instruction, and rubrics detailing the elements on which evaluation of the reports is based. The distinctive quality that characterizes the kit is careful coordination of the techniques offered in support of the writing tasks with the outcomes expected from the design work. It consists not just of advice to "write well", but of detailed instruction for writing techniques that foster highly usable design documentation [1].

The materials in the kit expand on the initial components laid out by a LaTeX outline: first they provide a detailed explanation of what and why they are included; then they present the techniques needed-the how-for managing the construction of the text and the presentation of informative, professional-level reports [3]. Throughout, the techniques discussed are specifically linked to both the inevitable assessment written documents receive in the classroom, as well as to the most likely type of scrutiny they face in industry settings [2].

\subsection{Delivering instructions}

The Kit has a three-tier design consisting of composing guidelines coordinated the template coordinated with the typesetting template, a series of workshops, and a targeted rubric for each of the four papers. First, the guidelines are established by examining the context in 
which the papers are produced. Specific conventions for the genres used are integrated with the components in the LaTeX outline, and the expectations laid out in the rubrics are factored into the catalogue of techniques that has been laid out. Four aspects needed for effective design reports - structural components, informative content management, text development, and visual formatting elements - are laid out as a reference chart to be consulted as composing moves forward. The guide is arranged in a 4- $\mathrm{x}-\mathrm{X}$ (6 for proposals/14 for preliminary reports) matrix, emphasizing that the composing categories are interrelated and apply to the work simultaneously, rather than being a sequence of rules. The guidelines are meant to be made available for students to use as they are researching the project and creating their design solutions, as well as when they write up the work as formal documentation.

The second tier of the kit consists of the presentation of the guidelines and associated writing advice in a workshop setting. These presentations are made to the whole class rather than in a tutorial and are not interactive, but they do focus on both the work the students are presently engaged in and the specific techniques that apply to the document required at a given stage. The emphasis in the PowerPoint slides is placed on the two central, and crucial, techniques for improving design report outcomes: managing the content instead of creating it, and developing the text in line with disciplinary expectations for how it forms part of the project.

These composing techniques are presented as part of the design process, involving organization and analysis for the first, the "Structuring by Content" part, and explanation and synthesis for the second, the "Developing Text" part. How and why the techniques in question are applied in the process is emphasized repeatedly, along with where it all goes. In addition to the academic applications developed by the communications instructor from extensive experience with teaching technical communication, the kit draws on industry examples from her background as a professional technical writer.

Considerable emphasis is placed on what can be called "Analytic Writing", an approach running directly counter to standard essay process. Our process advises the writer to ignore the standard advice to build from sentences into sentences to paragraphs, the technique that suits an essay meant to stimulate leisurely reflection rather than to prompt decision-making. Instead, we direct the writer to begin by categorizing the data/information/technical content that has been gathered, and then to employ the following sequence:

- identify the components of the design data as Sections, using genre conventions as a guideline;
- construct Paragraphs that make relevant assertions about the subject of any one section, including physical descriptions, explanation of operation, evidence of applications and implications for just that aspect; and

- formulate Sentences that are statements supporting the assertions by describing, explaining, giving evidence, and exploring implications.

Overall, the documentation aims not at creating content, but at managing engineering design work. The goal of a session is discussing the implications of the guidelines-how they affect the kind of data gathered; the way they insure that the data is turned into accurate, usable information; and when they can (almost) guarantee development of comprehensible and effective information.

The kit's contents are rounded out by a return to the question of the assessment the paper inevitably faces. For the Short Proposal, a Communications Evaluation Checklist to accompany the one-page grading sheet was created in discussion with the Teaching Assistants doing the grading. The checklist emphasizes meeting expectations for layout of the document, organization of the content, text development, and writing style and mechanics.

The terminology used here and later in the full-scale rubrics is familiar from standard essay practices, but is applied in a way that moves the student closer to the precisely targeted terms employed by the rubrics for the Preliminary and Final Design Reports. For the larger reports, a detailed section based on the guidelines and workshop was first added to the technical sections, increasing the scope of the rubric by a third.

For grading the preliminary reports, the rubric was discussed with the TAs to further clarify the assessment items; for the final report, the rubric was amended to place emphasis on the main problems encountered in the first iteration. An important effect of including the rubrics as a part of the writing kit, therefore, is to increase the amount and detail of information about the success, or not, of a report. But most importantly, it underscores the sometimes forgotten principle that outcomes are both first and last the measure of achievement in engineering work.

\section{Implementation}

The writing kit was delivered as three writing workshops, a research-and-reference session, and two LaTeX tutorials held during regular lecture periods throughout the semester. Each of the writing workshops was timed to fall approximately one week before a written deliverable was due. The first workshop was held in the second 
lecture period of the semester, and was intended to introduce students the expectations for writing in the course. It also had the specific goal of preparing them for the first writing assignment, a four-page project proposal due during the second week.

Since all deliverables were to be authored using LaTeX, an application unfamiliar to most students, the third lecture period was dedicated to a practical tutorial held in the computer lab rather than a lecture hall. Students were introduced to LaTeX and shown a demonstration of different processes and features, using the provided template for their proposal assignment as the primary example. They were also shown the steps for installing a software distribution of LaTeX that could be carried on a USB flash drive or placed on a laptop.

A research and referencing session was held to support of information research skill development, including critically evaluating sources and data. Through the just-in-time delivery of a customized, 80-minute, interactive workshop, students learned strategies for strategically searching the journal literature related to their projects, as well as for other, relevant resources such as prior art, standards, statistical information and government documents. For many students, Google is the first and last tool they use when seeking information. In this session, lead by the engineering librarian, they learned to critically evaluate the resources they retrieve through the web and to take their research further by using the key engineering databases and powerful search tools that are available to them through the library's paid subscriptions.

Many students use "RefWorks" as their bibliographic management software of choice. Provided and maintained by the University of Guelph Library, this software works seamlessly with many of the journal databases and other information research tools. Students quickly learn how to download their RefWorks citations into LaTeX's bibliographic tool, "BibTeX" so they can integrate the citations into their LaTeX documents. They have additional opportunities to further their knowledge of RefWorks by attending supplementary workshops on the software.

A second writing workshop, followed in turn by a second LaTeX tutorial, occurred in the week before the 40-page midterm report documenting the work of the group to date was due. A LaTeX template for the report, including heading examples was provided. A final writing workshop was held the week before the draft of a two page summary paper was due.

Workshops were also held for the course teaching assistants, with a focus on ensuring that the teaching assistants were familiar with the writing material and methods presented in class. The primary goal of these workshops was to ensure that the teaching assistants were marking the written portion of the course consistently, both with the writing expectations presented in the course, and with each other. These were timed to be after the writing workshops but before the due dates of major deliverables.

Finally, writing-specific support was provided for students outside of the regular classroom structure. Students were encouraged to meet with Dr. Hundleby specifically to discuss writing of their deliverables and to review drafts of written material. A strong minority of groups made use of this support at least once during semester, and anecdotal feedback was positive: students felt it improved their deliverables, and ultimately their mark. The course instructors also noted an increase in the number of requests to meet with them and review draft material, compared to previous semesters in which there had been no explicit emphasis on technical writing. For several groups, the improvement in written documents was noticeable, especially when comparing the final report submissions to draft material or the group's midterm report.

\section{Evaluation}

A small amount of anecdotal evidence suggests that timing of the workshops and tutorials had both positive and negative factors associated with them. Student attendance at the writing workshops and LaTeX tutorials was consistently higher than attendance for other course lectures, which were afflicted with a lecture time of 7:00 pm to 8:20 pm in the evening. Student attendance was consistently reduced at all lectures relative to prior years, with students commenting that the late timing of the lecture periods resulted in increased difficulty in attending regularly. Some students commented that these tutorials should have come earlier, more than one week before such a substantial document was due, however, for the majority of the students the week-before timing seemed reasonable.

Formal evaluation involved two surveys that are administered to ENGG*3100 students at the end of the semester. One is a generic university survey intended for global evaluation of courses and instructors that is of limited usefulness for evaluating specific initiatives within a course. Student opinions of the "overall" quality of the course and the instructors have remained broadly similar over the last three years. Slight improvement was noted for the most recent course offering; however, there were too many confounding variables in the course content and delivery to draw conclusions about specific aspects of the course.

The second survey is an anonymous three-page survey containing questions that seek feedback on specific 
aspects of the course. Each question consists of a written prompt, followed by a multiple-choice component asking students the degree to which they agree with the prompt, and adding an area for written comments related to it. The survey is distributed to students during a series of final design reviews that are held during the last week of classes.

The survey has been administered in ENGG*3100 in 2008, 2009 and 2010, with the majority of questions remaining the same and allowing comparison between years. The 2010 survey was modified to include requests for feedback on the writing instruction and support within the course. Response rates always exceeded $80 \%$ of students, suggesting the surveys represent a true sample of student opinions. Of the surveys returned, more than $90 \%$ included responses to all multiple choice questions and at least some written comments. Although the survey did not specifically ask about the importance of learning and practising engineering writing skills, many students included written comments noting the importance of technical writing as an engineering skill.

The question that asked about the value of the writing workshops received mixed responses. The variation in the written comments suggests that students interpreted the question several different ways: some opinions were set in the context of Dr. Hundleby's lecturing style, some focussed on the value of the workshops only, and some related to the overall support for writing in the course. There was general agreement that including support for writing was important in a course. A number of students did not like having to use LaTeX citing the difficulty of learning a new program when they felt an existing tool such as Word to be sufficient. A few also noted that LaTeX is rarely used in industry and therefore felt it was not a useful skill to spend time learning because it would not be needed by future employers.

Several students commented that the report format should be "open to different writing styles" and should acknowledge there is "more than 1 way to write a good engineering report." Students seem to feel that they should be allowed more creativity in the presentation of their written work, and it may well be that students felt that they had to follow a large number of rules and spend unreasonable amounts of time on report writing. A telling feature of the typical student relationship to the writing support offered was that, when a template for a document is provided, they have difficulty deciding when it is appropriate to modify the template (e.g. change provided headings) and when it is not (e.g. eliminate required elements in the document).

Interestingly, one student commented on "never [having] been asked to write a report like this before"- is, a full-length formal report with detailed expectations about presentation of material-and wished for more "warning" that expectations for writing quality would be high in the course. The instructors note that the expectations for deliverables were presented in the course outline, and repeated during practically every lecture and individual meeting with students. The authors believe that such comments indicate that technical writing is a skill needing considerable development effort and practice throughout the curriculum, with further work in the area during curriculum development paramount. A curriculum that designates one or a few courses as the place where "writing" is learned while not using or practising those skills in other courses will not result in graduating students who have a mastery of written engineering communication upon graduation.

\section{Conclusions}

The Writing Kit for Engineering Design Reports draws on three central concepts to create an effective introduction of targeted writing into an engineering design course setting. The first is that it contextualizes the work, grounding it in the expectations of the discipline of engineering and the requirements of the aspect of engineering that the design work addresses. The second is that the instruction replaces global, all-purpose advice with the techniques that enable students to produce the kind of analytic writing demanded by scientific and technical work. The third, and probably the most important, concept is that of enlisting assessment strategies to furnish a basis for explain the writing techniques that will assist in a successful, usable outcome. While nothing will absolutely guarantee success, the approach outlined here forms a promising beginning for integrating specific writing instruction into engineering work. The writing kits is available for download from the University of Guelph library repository http://atrium.lib.uoguelph.ca/

\section{References}

[1] M. N. Hundleby, and J. Allen, eds., Assessment in Technical and Professional Communication, Baywood Publishing Company, Garden City, L. I., 2010.

[2] M. Lynch, Scientific Practice and Ordinary Action, Cambridge University Press, Cambridge, UK, 1993.

[3] D. A. Winsor, Writing like an engineer: A rhetorical education, Lawrence Erlbaum Associates, Rahway, NJ, 1996. 